|| Print ISSN: 2589-7837 || Online ISSN: 2581-3935 || International Journal of Medical Science and Diagnosis Research (IJMSDR)

Available Online at www.ijmsdr.com

NLM (National Library of Medicine ID: 101738824)

\title{
SIX CANALLED MAXILLARY FIRST MOLARS. A CASE REPORT
}

Dr. Ruchika Gupta Dewan ${ }^{1}$, Dr. Anchal Aggarwal ${ }^{2}$, Dr. Mandira Gulati ${ }^{3}$, Dr. Unnavi Chauhan ${ }^{4}$

${ }^{1}$ MDS, Professor, Dept. Of Conservative Dentistry and Endodontics ${ }_{L}$ I.T.S. Dental College, Hospitals and Research Centre ${ }_{\llcorner} 47$, Knowledge Park IIILGreater Noida, UP

${ }^{2,3,4}$ Student, Dept. of Conservative Dentistry and Endodontics, I.T.S. Dental College, Hospitals and Research Centre, 47, Knowledge Park III, Greater Noida, UP

Conflicts of Interest: Nil

Corresponding author: Dr. Ruchika Gupta

DOI: https://doi.org/10.32553/ijmsdr.v4i11.713

\section{Abstract:}

Aim: This Case Report describes two rare cases of clinical management of maxillary first molars with six root canals with special reference to radiographic interpretation and diagnosis.

Background: The study reports 2 clinical cases of endodontic treatment of maxillary first molars with six root canals.

Case Description: The access cavity was prepared using a slow speed round bur. The teeth were diagnosed with irreversible pulpitis and then anesthetized for an endodontic access cavity preparation. Clinical evaluation of the internal anatomy revealed 3 principle root canal systems: mesiobuccal (MB), distobuccal (DB), and palatal in each tooth. The working length was determined and the radiographs were taken for all the roots i.e mesiobuccal, distobuccal and palatal separately after placing instruments in each. The cleaning and shaping was performed using ProTaper Universal rotary instruments. Irrigation between each instrument was done. The canals were dried and obturation was performed using cold lateral compaction of gutta-percha and a resin-based sealer. The teeth were then restored with a posterior composite restoration. The patients were advised a full-coverage crown.

Conclusion: A good knowledge of tooth morphology, careful interpretation of angled radiographs, proper access cavity preparation and a detailed exploration of the interior of the tooth is needed to ensure a proper endodontic treatment.

Clinical Significance: Prevalence of teeth with such complex internal anatomy is a rare anomaly. However, a sound knowledge of the internal anatomy can aid in its adequate management.

Keywords: maxillary molar, six canals, altered anatomy

\section{Introduction:}

\section{Background:}

A clear understanding of the human teeth anatomy is a prerequisite to achieving proper access cavity preparation, cleaning, disinfection and obturation of the pulp space. ${ }^{1,2}$ A thorough knowledge of the root canal anatomy, its variations, the presence of additional roots, and unusual root canal morphology is essential, as it determines the successful outcome of endodontic treatment ${ }^{3}$. Numerous factors contribute to the variations found in root canal studies including ethnicity, age, gender, and study design. ${ }^{4,5}$ The morphology of the maxillary first molar has been extensively studied. Additional canals have been found in distobuccal and palatal roots also. Other rare variations include presence of two roots, single root, four roots, or C-shaped root canal system. ${ }^{6}$
It is generally accepted that three roots and four canals in the maxillary first molar should be regarded as the rule rather than the exception ${ }^{7}$. The presence of the fourth canal is usually located in the mesiobuccal (MB) root which ranges from $18.6 \%$ to $96.1 \%{ }^{8,9}$ Additional canals were reported in the distobuccal (DB) and palatal (P) roots. Case reports with five, ${ }^{10}$ six, ${ }^{11}$ seven, $^{12}$ and even eight canals were reported. ${ }^{13}$ Currently, technological advances have been developed, and different techniques have been introduced to facilitate the assessment of internal anatomic variations of root canals. The purpose of the present case report is to highlight the endodontic management of two rare cases of maxillary first molars with three roots and six canals each.

\section{Case Description:}

The study reports two clinical cases of endodontic treatment of maxillary first molars with three six root canals respectively. 


\section{Case Report 1}

A 48-year-old female patient reported to the Department of Conservative Dentistry and Endodontics with the chief complaint of pain in the left upper back tooth region. The pain was continuous in nature and aggravated on heat stimulation. The patient's medical history was non-contributory. Clinical examination revealed a deep carious lesion in the left maxillary first molar which was tender on percussion. Electric Pulp Testing gave no response. On thermal test (cold test) a delayed lingering response was elicited. The radiographic examination revealed a radiolucent lesion on the distal aspect of the crown extending to the pulp in relation to \#26 (maxillary left first molar) (Fig.1a), with an abrupt loss of radiolucency in the pulp canal. A diagnosis of irreversible pulpitis was made, endodontic treatment of the involved tooth was planned and an informed consent was taken from the patient.

The tooth was then anesthetized with a solution of $2 \%$ lidocaine with 1:100000 epinephrine by way of Posterior Superior Alveolar Nerve Block, Greater Palatine Nerve Block and Buccal infiltration. A conventional endodontic access cavity was prepared. Clinical evaluation of the internal anatomy revealed 3 principle root canal systems: mesiobuccal (MB), distobuccal (DB), and palatal. After probing with a DG 16 endodontic explorer, small hemorrhagic points were noted $2 \mathrm{~mm}$ palatal to the $\mathrm{MB}$ and $\mathrm{DB}$ canals. As the dentin that was occluding the orifice of the palatal canal was removed, a second palatal canal was also identified. There seemed to be 2 distinct orifices in all the roots (Fig.1b).The working length was determined with the help of $\mathrm{K} \& \mathrm{H}$ files and the instrument was kept $0.5 \mathrm{~mm}$ short of radiographic apex. After the establishment of working length an initial glide path was established using hand file. Radiographs were taken for all the roots i.e mesiobuccal, distobuccal and palatal separately after placing instruments in each (Fig.1c,1d,1e).

The cleaning and shaping was performed using ProTaper nickel-titanium rotary instruments (Dentsply Maillefer, Switzerland). Irrigation between each instrument was done using $2.5 \%$ sodium hypochlorite solution and a final flush of $17 \%$ EDTA. A sterile cotton pellet and an interim restoration of Cavit (3M Espe, Seefeld, Germany) was placed in the pulp chamber to seal the access cavity. In the subsequent visit the canals were dried and obturated using cold lateral compaction using gutta-percha points (Dentsply Maillefer) and a resin-based sealer (AH Plus, Maillefer, Dentsply, Konstanz, Germany). The tooth was then restored with a posterior composite restoration (Fig. 1f). Two months later the patient was reviewed and the tooth was asymptomatic. The patient was advised a full-coverage restoration.

\section{Case Report 2}

A 37-year-old male patient reported to the Department of Conservative Dentistry and Endodontics with the chief complaint of pain in the left upper back tooth region. The chief complaint of the patient was pain in the left maxillary first molar tooth with a history of multiple attempted restorations. Clinical examination revealed a temporary restoration which was also tender on percussion. The pain was continuous in nature and aggravated on application of heat. On thermal test (cold test) a delayed response was elicited. After clinical and radiographic examination, it was diagnosed as a case of irreversible pulpitis. The radiographic examination revealed a restorative material in relation to \#26 (maxillary left first molar), below which could be seen a radiolucent lesion extending to the pulp (Fig.2a). A diagnosis of irreversible pulpitis was made and the treatment plan of Root Canal Treatment was explained to the patient.

The tooth was then anesthetized with a solution of $2 \%$ lidocaine with 1:100000 epinephrine by way of Posterior Superior Alveolar Nerve Block, Greater Palatine Nerve Block and Buccal infiltration. An endodontic access cavity was prepared. Clinical evaluation of the internal anatomy revealed 3 principle root canal systems: mesiobuccal (MB), distobuccal (DB), and palatal. Further troughing with Ultrasonics revealed six canal orifices separately (Fig.2b).The working length was determined with the help of $\mathrm{K} \& \mathrm{H}$ files and the instrument was kept $0.5 \mathrm{~mm}$ short of radiographic apex. Radiographs were taken for all the roots i.e distobuccal, palatal and mesiobuccal separately after placing the $\mathrm{H}$ and $\mathrm{K}$ flies in each (Fig.2c,2d,2e).

The cleaning and shaping was performed using ProTaper nickel-titanium rotary instruments (Dentsply Maillefer, Switzerland). Irrigation between each instrument was done using $2.5 \%$ sodium hypochlorite solution and with a final flush of $17 \%$ EDTA. A sterile cotton pellet and an interim restoration of Cavit (3M Espe, Seefeld, Germany) was placed in the pulp chamber to seal the access cavity. At the subsequent visit the canals were dried and obturated using cold lateral compaction of gutta-percha (Dentsply Maillefer) and a resin-based sealer (AH Plus, Maillefer, Dentsply, Konstanz, Germany) The access cavity was then sealed with a permanent restorative material (composite resin (Z250, 3M ESPE, Sumar'e, SP, Brazil)) (Fig. 2f). 


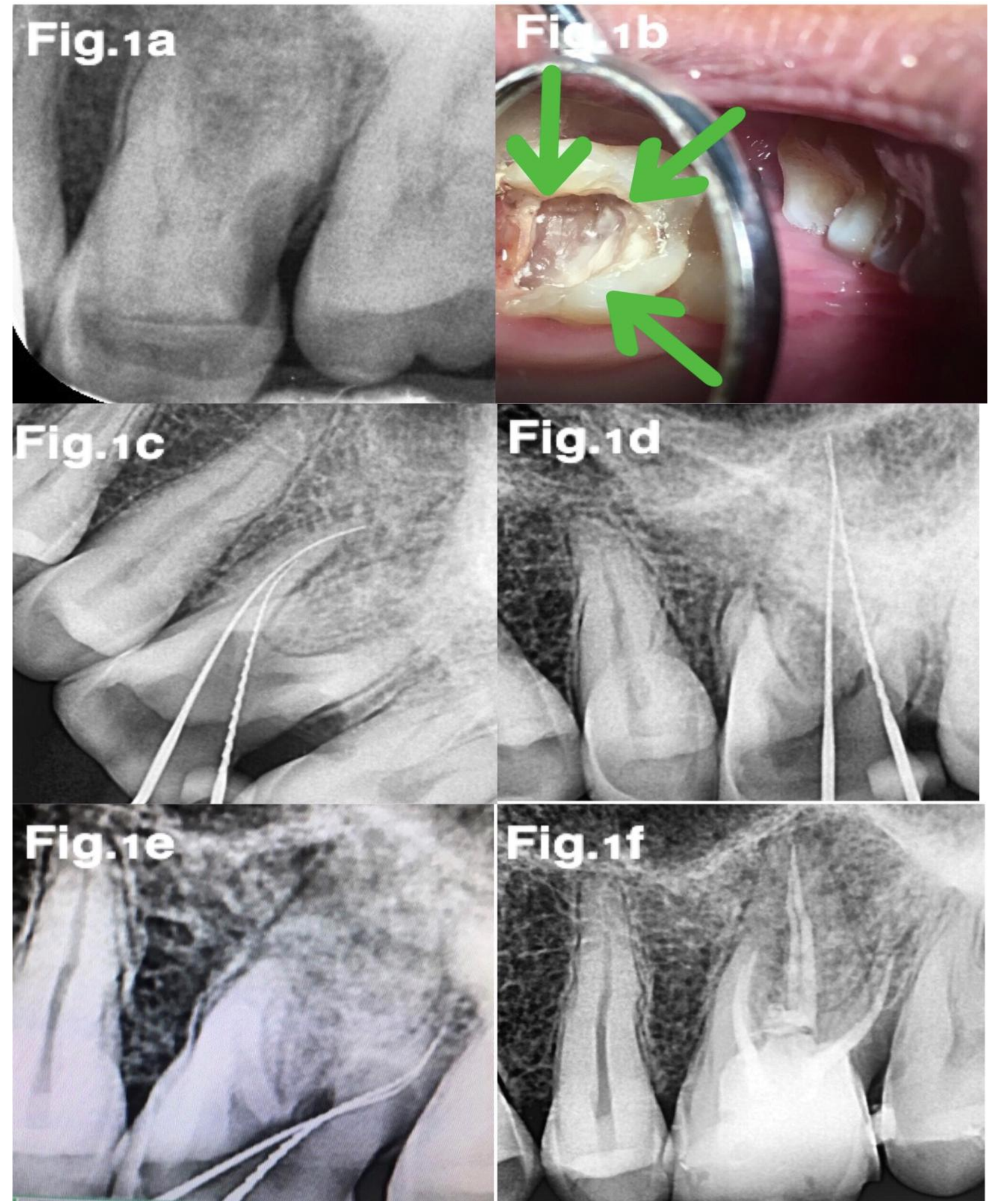

Fig.1a: Pre-operative Radiograph

Fig.1b: Intra-oral Photograph

Fig.1c: Working Length Radiograph of mesial canals

Fig.1d: Working Length Radiograph of palatal canals

Fig.1e: Working Length Radiograph of distal canals

Fig.1f: Post-operative Radiograph 


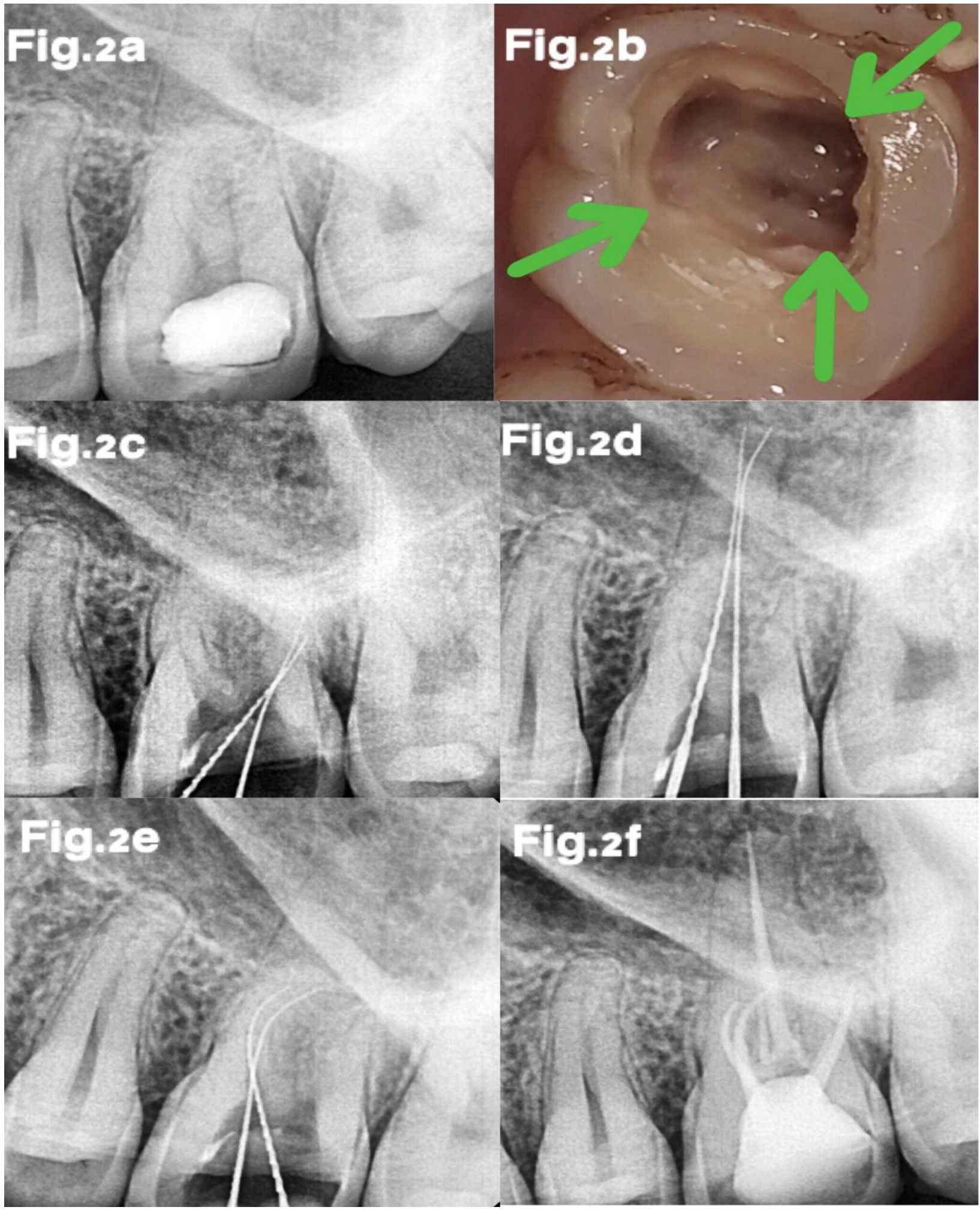

Fig.2a: Pre-operative Radiograph

Fig.2b: Intra-oral Photograph

Fig.2c: Working Length Radiograph of distal canals

Fig.2d: Working Length Radiograph of palatal canals

Fig.2e: Working Length Radiograph of mesial canals

Fig.2f: Post-operative Radiograph 


\section{Discussion:}

An accurate diagnosis of the anatomy of the root canal system is a prerequisite for successful endodontic treatment. ${ }^{14,15}$ Therefore, the radiographic signs that demonstrate the presence of anatomical variations must be considered an important condition when planning the tooth treatment. ${ }^{16}$ Detection of aberrant anatomy can be done by: In vivo Methods: Loupes, Operating Microscope, Dyes, Champagne Bubble Test, Following the dentinal map, Anatomy of the crown and In vitro Methods: Direct observation, Microscopic observation, Macroscopic sectioning, Microscopic sectioning, Dyes, Filling \& decalcification, Filling \& clearing, Radiography, Contrasting media (Hypaque), Cone beam Tomography. ${ }^{17,18}$

The maxillary first molar is considered one of the most complex teeth in the dental arch. Anatomical variations are commonly observed in maxillary first molar. The incidence of second mesiobuccal (18.6\%-96.1\%), second distobuccal (1.6\%-9.5\%), and mesiopalatal (56.8\%) and the prevalence of distopalatal (1.7\%) canals have been reported in various studies. ${ }^{19}$ Proper access opening and modifying the shape of the access to approach all orifices is a key to success in identifying and negotiating unusual anatomy of root canals. In the present case reports, the conventional triangular access was modified to trapezoidal to improve access to the additional canals. ${ }^{20,21}$ Recently, the clinical use of microscopy has been emphasized as an aid in the localization of calcified canals and in the identification of anatomical variations. ${ }^{22}$ This tool can be especially useful when it is possible to identify independent entrances for each canal. ${ }^{23}$

Buhrley et $\mathrm{al}^{24}$ concluded that when the maxillary first molars were considered separately, the frequency of MB2 canal detection for the microscope, dental loupes, and no magnification groups were $71.1 \%, 62.5 \%$, and $17.2 \%$, respectively. Baldssari-Cruz et al ${ }^{25}$ evaluated 39 maxillary molars in vitro and concluded that the surgical operating microscope did aid in the identification of mesiolingual canal orifices. In their study, they detected mesiolingual canals in 20 teeth with unaided vision, but the presence of mesiolingual canals was located in additional 12 teeth using the surgical operating microscope.

In this case, however, we did not feel the need for any objective analytical tool, such as CT and spiral or helical CT, to ascertain root canal morphology, because there were no doubtful circumstances in either radiographs of different angulations or examination of the floor of the pulp chamber. Furthermore, such equipment may not always be present in routine clinical practice, and the patient can be exposed to unwarranted radiation. ${ }^{26}$ The simultaneous occurrence of double canal systems in all roots of maxillary first molar is an unusual finding. Therefore, a thorough knowledge of root and root canal morphology and a good anticipation of their possible morphologic variations are essential and will help to reduce endodontic failure caused by incomplete root canal preparation and obturation. Thus, it is important to be conscious to variations from the expected and to use all the armamentaria available to locate and treat the entire root canal system.

\section{Clinical Significance:}

This paper presents the management of two patients who presented with the uncommon anatomy of six root-canalled maxillary first molars. Clinicians should be constantly on the lookout for 'occult' anatomy. Access cavity refinements may be required for stress-free entry to complex anatomy. A thorough analysis of angled radiographs, conscientious exploration of the floor of the pulp chamber, a good knowledge of the anatomical description with possible variation of the canal system and sufficient time for treatment are necessary to achieve a successful outcome in root canal therapy.

\section{References:}

1. Sulaiman A, Dosumu $O$ and Amedari M. Maxillary first premolar with three root canals: A case report. Ann Ibd. Pg. Med 2013. Vol.11, No.2 105-108

2. Dax A, Pravin K. Radiculous premolar. Endodontology 2011;23(2): 95-97

3. Malagnino V, Gallottini L, Passariello P. Some unusual clinical cases on root anatomy of permanent maxillary molars. J Endod. 1997 Feb 23(2):127-8.

4. Gupta S, Sinha D, Gowhar O, Tyagi S, Singh N, Gupta S. Root and canal morphology of maxillary first premolar teeth in north Indian population using clearing technique: An in vitro study. Journal of Conservative Dentistry | May-Jun 2015 | Vol 18 | Issue 3

5. Mittal S, Kumar T, Mittal S, Sharma J. Mandibular premolars with aberrant canal morphology: An endodontic challenge. J Conserv Dent 2014;17:491-4.

6. Cleghorn BM, Christie WH, Dong CC. Root and root canal morphology of the human permanent maxillary first molar: A literature review. J Endod 2006;32: 813-21.

7. Pineda F, Kuttler Y. Mesiodistal and buccolingual roentgenographic investigation of 7,275 root canals. Oral Surg Oral Med Oral Pathol1972;33:101-10.

8. Baratto Filho F, Zaitter S, Haragushiku GA, de Campos EA, Abuabara A, Correr GM. Analysis of the internal anatomy of maxillary first molars by using different methods. J Endod2009;35:337-42. 
9. Kulild JC, Peters DD. Incidence and configuration of canal systems in the mesiobuccal root of maxillary first and second molars. J Endod1990;16:311-7.

10. Shetty K, Yadav A, Babu VM. Endodontic management of maxillary first molar having five root canals with the aid of spiral computed tomography. Saudi Endod J 2014;4:149-53.

11. Bond JL, Hartwell G, Portell FR. Maxillary first molar with six canals. J Endod1988;14:258-60.

12. Badole GP, Warhadpande MM, Shenoi PR, Lachure C, Badole SG. A rare root canal configuration of bilateral maxillary first molar with 7 root canals diagnosed using cone-beam computed tomographic scanning: $A$ case report. J Endod2014;40:296-301.

13. Kottoor J, Velmurugan N, Surendran S. Endodontic management of a maxillary first molar with eight root canal systems evaluated using cone-beam computed tomography scanning: A case report. J Endod 2011; 37:715-9.

14. Relvas J B F, Carvalho $F M$, Marques A A F, SponchiadoJr E C, Garcia L F R. Endodontic Treatment of Maxillary Premolar with Three Root Canals Using Optical Microscope and NiTi Rotatory Files System. Hindawi Publishing Corporation Case Reports in Dentistry Volume2013,ArticleID710408.

15. C.Aguiar,D.Mendes,A.C^amara, andJ.Figueiredo, "Endo dontictreatmentofamandibularsecondpremolarwithth reeroot canals," Journal of Contemporary Dental Practice, vol. 11, no. 2, pp.78-84,2010.

16. F. J. Vertucci, "Root canal morphology and its relationship to endodontic procedures" Endodontic Topics,vol.10,no.1,pp.3-29,2005.
17. Kumar T, Sanap A, Bhargava K, Aggarwal S,Kaur G, Patil P. Management of A Maxillary Premolar With Three Roots: A Case Report. Int J Cont Med Res. 2015;2(1):136-140.

18. Nallapatti S. Aberrant root canal anatomy: a review. Endod bulletin, 2007; 18: 50-62.

19. Thomas RP, Moule AJ, Bryant R. Root canal morphology of maxillary permanent first molar teeth at various ages. Int Endod J 1993;26:257-67.

20. Kaushik M, Mehra N. Maxillary first molars with six canals diagnosed with the aid of cone beam computed tomography: A report of two cases. Case Rep Dent 2013;2013:406923

21. Y.-Y. Lee, P.-Y. Yeh, S.-F. Pai, and S.-F. Yang, "Maxillary first molar with sixcanals,"Journal of Dental Sciences, vol. 4, no. 4, pp.198-201,2009.

22. Kontakiotis EG, Tzanetakis GN. Four canals in the mesial root of a mandibular first molar. A case report under the operating microscope. Aust Endod J 2007; 33:84-88.

23. Scarparo RK, Pereira L, Moro D, Gründling G, Gomes M, Grecca FS. Morphologic Variations of Maxillary Molars Palatal Root and the Importance of Its Knowledge for Endodontic Practice: A Case Series. J Contemp Dent Pract 2011;12(2):138-142.

24. Buhrley LJ, Barrows MJ, BeGole EA, Wenckus CS. Effect of magnification on locating the MB2 canal in maxillary molars. J Endod 2002;28:324-7

25. Ferguson D B, Kjar KS, Hatwell GR SThree canals in the mesiobuccal root of a maxillary first molar: A case report. J Endod 2005;31:400-2.

26. Maggiore F, Jou YT, Kim S. A six-canal maxillary first molar: Case report. Int Endod J 2002;35:486-91. 